\title{
ESTUDOS BIOLÓGICOS DE COCHONILHAS DO GÊNERO PLANOCOCCUS (HEMIPTERA: PSEUDOCOCCIDAE) EM DIFERENTES HOSPEDEIROS
}

\author{
L.R.B. Correa ${ }^{1}$, B. Souza ${ }^{2}$, L.V.C. Santa-Cecília ${ }^{3}$, E. Prado ${ }^{4}$
}

${ }^{1}$ Universidade Estadual Paulista, Faculdade de Ciências Agrárias e Veterinárias, Departamento de Fitossanidade, Via de Acesso Prof. Paulo Donato Castellane, s/nº, CEP 14884-900, Jaboticabal, SP, Brasil. E-mail: robertaento@yahoo.com.br

\section{RESUMO}

Este trabalho teve por objetivo estudar o desenvolvimento de populações de cochonilhas do gênero Planococcus em diferentes espécies vegetais, visando à verificação de especificidade hospedeira. A cochonilha Planococcus minor foi obtida de plantas de cacau (Theobroma cacao L.) e de café (Coffea canephora Pierre), enquanto Planococcus citri foi obtida de plantas de citros (Citrus sinensis L. Osbeck). Ovos foram transferidos individualmente para placas de Petri contendo discos foliares de cacau (T. cacao cultivar Catongo), café (Coffea arabica L. cultivar Mundo Novo) e citros (C. sinensis cultivar Pêra Rio), utilizando-se 60 repetições. Esses discos foliares foram mantidos sobre uma lâmina de ágar-água a 1\%, com a superfície abaxial voltada para cima. As placas foram vedadas com filme plástico de PVC e mantidas em câmaras climatizadas reguladas a $25 \pm 1^{\circ} \mathrm{C}, 70 \pm 10 \%$ UR e 12 horas de fotofase. Para as cochonilhas oriundas de cacaueiro, o substrato citros prolongou o desenvolvimento ninfal dos machos (26,0 dias). Para os insetos provenientes de cafeeiro, o substrato café proporcionou o menor período ninfal de fêmeas e machos (19,2 e 21,3 dias, respectivamente) e maior longevidade das fêmeas (59,1 dias). Os maiores índices de mortalidade foram obtidos quando as cochonilhas foram criadas no substrato cacau, independente da planta hospedeira da qual foram originalmente coletadas, e quando mantidas em citros, a partir de espécimes coletados em plantas de café. Há maior preferência alimentar de $P$. citri e $P$. minor para o substrato café, independente do hospedeiro de origem, o que indica uma especificidade hospedeira parcial.

PALAVRAS-CHAVE: Cochonilha-branca, biologia, especificidade alimentar.

\section{ABSTRACT}

BIOLOGIC STUDIES OF MEALYBUGS OF THE GENUS PLANOCOCCUS (HEMIPTERA: PSEUDOCOCCIDAE) ON DIFFERENT HOSTS. This work was carried out to study the development of populations of the genus Planococcus in different plant species, aiming at the verification of host specificity. The mealybugs Planococcus minor were obtained from cocoa plants (Theobroma cacao L.) and coffee crop (Coffea canephora L.), and Planococcus citri from citrus seedlings (Citrus sinensis L. Osbeck). Single eggs were transferred to Petri dishes containing leaf discs of cocoa (T. cacao cultivar Catongo), coffee (Coffea arabica L. cultivar Mundo Novo) and citrus (C. sinensis cultivar Pêra Rio). They were maintained on a water-agar slide at $1 \%$, with the abaxial surface turned upwards. The dishes were sealed with a PVC plastic film and kept in climatized chambers regulated at $25 \pm 1^{\circ} \mathrm{C}$, $70 \pm 10 \% \mathrm{RH}$ and 12 hours of photophase. For the mealybugs coming from cocoa plants, the citrus substrate prolonged the nymphal development of the males (26.0 days). In the nymphal period of females and males coming from coffee plants, the coffee substrate allowed a shorter duration of that period (19.2 and 21.3 days, respectively) and, in addition to having providing a longer longevity to the females (59.1 days). The highest rates of mortality were obtained when they were reared on the cocoa substrate, regardless of the host plant from which they were originally collected, and when kept on citrus, for insects collected on coffee plants. P. citri and P. minor have higher preference for coffee substrate regardless of the original host, thus showing partial host specificity.

KEY WORDS: Citrus-mealybugs, biology, specificity.

${ }^{2}$ Universidade Federal de Lavras, Departamento de Entomologia, Lavras, MG, Brasil.

${ }^{3}$ IMA/EPAMIG/URESM-EcoCentro, Laboratório de Controle Biológico de Pragas, Lavras, MG, Brasil.

${ }^{4}$ EPAMIG/URESM-EcoCentro, Laboratório de Controle Biológico de Pragas, Lavras, MG, Brasil. 


\section{INTRODUÇÃO}

As cochonilhas constituem um dos principais grupos de insetos-praga em diversos sistemas de produção, infestando plantas silvestres e cultivadas, seja em campo ou em cultivos protegidos (CLAPS; TERÁn, 2001; SANTA-Cecília; SOUZA, 2005).

Muitos aspectos da vida do inseto, tais como comportamento, fisiologia e ecologia estão, de alguma maneira, relacionados com a qualidade equantidade do alimento utilizado, tornando fundamentais as relações tróficas entre insetos e plantas (SLANSKY JUNIOR; RODRIGUEZ, 1987).

Os insetos fitófagos dependem das plantas para sobreviver e estão sujeitos a todas as alterações que resultam das interações entre elas e o meio ambiente. Entretanto, essa associaçãoíntima não afeta somentea eles, pois uma infestação intensa de insetos não passa despercebida pela planta (OsBORNE, 1973). Para minimizar oataque desses fitófagos, as plantas desenvolveram estruturas físicas e/ ou substâncias químicas que causam efeitos deletérios aos insetos não adaptados a se alimentar das mesmas (Pizzamiglio, 1991).

Por outro lado, muitos insetos desenvolveram mecanismos para manipular essas defesas de suas plantas preferidas ou mesmo utilizá-las em benefício próprio, como atraentes e estimulantes da alimentação (PANIZZI; PARRA, 1991). Desse modo, as substâncias secundárias, que inicialmente foram selecionadas pelas plantas para sua defesa, tornaramse cairomônios para insetos, que desenvolveram mecanismos para sobrepujá-las, conduzindo para uma especificidade hospedeira (HAGEN et al., 1984).

SLANSKY JUNIOR; RODRIGUEZ (1987) propuseram que um grupo ou espécie de planta hospedeira apresenta características físicas, químicas e fenológicas que podem ser relacionadas com os caracteres fisiológicos e comportamentais do herbívoro. De acordo com BERNAYS; CHAPMAN (1994), os insetos sugadores comumente exibem adaptações morfológicas e fisiológicas associadas com seu modo particular de alimentação, em tecidos específicos e, dessa forma, a maioria apresenta especificidade para espécies hospedeiras.

Algumas espécies de Planococcus (Hemiptera: Pseudococcidae) apresentam especificidade hospedeira para família de plantas, e até mesmo gênero. As espécies desse gênero foram divididas em grupos, por meio de características morfológicas e suas plantas hospedeiras. Assim, espécies do grupo-dendrobii, por exemplo, são específicas de Orchidaceae, e outras, apesar de terem uma ampla gama de hospedeiros, apresentam preferências distintas. A espécie Planococcus citri (Risso, 1813), por exemplo, que é considerada polífaga, não é frequentemente encontrada em campos de videiras, enquanto Planococcus ficus (Signoret, 1869), comum em videiras, raramente é encontrada em citros (Cox, 1989).
A ocorrência de espécies crípticas entre cochonilhas é bastante comum (Miller; Kosztarab, 1979), como verificado em Planococcus citri (Risso, 1813) e Planococcus minor (Maskell, 1897), o que tem dificultado a identificação específica desses insetos com base em caracteres morfológicos externos. Além disso, essas espécies podem ocorrer na mesma planta hospedeira, como foi relatado por SANTA-CECílIA et al. (2002) em cafeeiros.

Nessas situações, a especificidade alimentar constitui-se em um caráter taxonômico que pode contribuir para o esclarecimento dessa questão.Sendo assim, devido à considerável variação intraespecífica dos caracteres morfológicos utilizados para a identificação dessas cochonilhas, é frequentemente difícil determinar se uma forma difundida e variável representa uma ou várias espécies, as quais podem permanecer não distinguidas até que discrepâncias emsua biologia ou ecologia sejam notadas (Cox, 1983).

Nur (1978) sugeriu que, pelo menos em Pseudococcidae, a diferenciação morfológica desenvolve mais lentamente em relação às características que afetam oisolamento reprodutivo. Diferenciações na composição de feromônios de machos de espécies crípticas sugerem que a conservação morfológica podeser um fenômeno bem difundido em Coccoidea (Miller; Kosztarab, 1979).

Devido à cochonilha $P$. citri ser muito similar à P. minor, esta foi identificada incorretamente como P. citri até a publicação de Cox; FREESTON (1985), que separaram as duas espécies com o uso do método de notas (score), pois as chaves dicotômicas tradicionais mostraram-se infactíveis. Uma vez que os caracteres utilizados na identificação apresentam uma variação considerável em função das condições ambientais sob as quais os espécimes são criados, os valores dos caracteres considerados se sobrepõem atécerto ponto entre as duas espécies, mas eles são característicos e determinantes para elas (Cox, 1983).

Assim sendo, este trabalho teve por objetivo estudar o desenvolvimento de populações de cochonilhas do gênero Planococcus em diferentes espécies vegetais, visando à verificação de especificidade hospedeira.

\section{MATERIAL E MÉTODOS}

Colônias de Planococcus spp. utilizadas nos experimentos foram obtidas de plantas de cacau (Theobroma cacao L. cultivar Comum) cultivadas em casa de vegetação em Lavras, MG, em lavouras de café (Coffea canephora Pierre cultivar Conilon) localizadas no Município de Cachoeiro do Itapemirim, ES, e em mudas de citros (Citrus sinensis L. Osbeck cultivar Valência) cultivadas em casa de vegetação, em Piracicaba, SP. Para a confirmação genérica utilizou-se a chave dicotômica de Cox (1989) e para 
a determinação específica foram realizadas análises morfológicas em microscopia óptica de acordo com a metodologia proposta por COx; FREESTON (1985). As populações provenientes de plantas de cacau e café foram identificadas como $P$. minor e as procedentes de plantas de citros como $P$. citri.

Ovos provenientes dessas colônias foram utilizados na instalação dos bioensaios, sendo transferidos individualmente para placas de Petri $(5 \mathrm{~cm}$ de diâmetro) contendo discos foliares de cacau ( $T$. cacao cultivar Catongo), café (Coffea arabica L. cultivar Mundo Novo) e citros (C. sinensis cultivar Pêra Rio). Esses discos foliares ( $4 \mathrm{~cm}$ de diâmetro) foram mantidos sobre uma lâmina de aproximadamente $5 \mathrm{~mm}$ de ágar-água a 1\%, com a superfície abaxial voltada para cima. As placas foram vedadas com filme plástico de PVC e mantidas em câmaras climatizadas a $25 \pm 1^{\circ} \mathrm{C}, 70 \pm 10 \%$ URe 12 horas de fotofase. Quando os discos foliares iniciavam o processo de amarelecimento as placas eram substituídas.

As cochonilhas foram observadas em intervalos de 24 horas ao longo de todo seu período de vida, avaliando-se o número de ínstares, a duração e a mortalidade no período ninfal, a longevidade e o ciclo de vida de machos e fêmeas. Como não há diferenciação sexual evidente no início do desenvolvimento ninfal, as repetições foram constituídas por indivíduos com sexo não conhecido.

O delineamento experimental foi inteiramente casualizado com 60 repetições, sendo cada repetição constituída por um inseto encerrado em uma placa, e os tratamentos, pelos substratos alimentares.

Os dados da duração do período ninfal, longevidade e ciclo de vida foram submetidos à análise de variância(ANAVA) eas médias comparadas pelo teste de Tukey a 5\% de significância, com dados transformados em $\sqrt{x}$. Para o cálculo da sobrevivência foi utilizado o teste de Qui-quadrado a 5\% de significância. Nesse cálculo não se considerou o sexo dos insetos, pois alguns morreram no primeiro ínstar e início do segundo, ocasiões em que ainda não é evidente a diferenciação sexual, obtendo-se a mortalidade geral.

\section{RESULTADOS E DISCUSSÃO}

Como já relatado para a maioria dos pseudococcídeos (GARCia et al., 1992; MALleSHaiAH et al., 2000; SANTA-CeCília et al., 2004; Correa et al., 2005), o desenvolvimento ninfal de Planococcus spp. proveniente de todos os hospedeiros constou de quatro ínstares para os machos, sendo o terceiro e quarto passados dentro de um casulo, e de três ínstares para as fêmeas.

Duranteoperíodoexperimental, asfêmeas adultas não apresentaram reprodução por partenogênese e, embora algumas delas tenham iniciado a construção do ovissaco, não houve postura. Essas observações discordaram daquelas realizadas por MALLESHAIAH et al. (2000), na Índia, que constataram reprodução sexuada e partenogenética em $P$. citri.

De uma maneira geral, o número de machos foi superior ao defêmeas, com restriçãoa dois tratamentos (cacaueiro/cacau e cafeeiro/café), o que difere dos resultados de MALLESHAiAh et al. (2000) e CORREA et al. (2005)queconstataramumnúmerosuperior defêmeas, embora CORREA (2006) tenha verificadouma quantidade de machos ligeiramente maior que a de fêmeas.

As cochonilhas fêmeas oriundas de cacaueiro apresentaram um desenvolvimento ninfal semelhante em todos os substratos alimentares (Tabela 1). Os valores constatados para esse período foram superiores aos encontrados por MAITY et al. (1998), quando estudaram a cochonilha $P$. minor criada em tubérculos de batata (17,1 dias) e frutos de abóbora (17,8 dias), entretanto, foram inferiores ao obtido quando criada em inhame (31,7 dias). Para os machos, o período ninfal foi influenciado pelos substratos alimentares, sendo que as ninfas que se desenvolveram em citros apresentaram maior duração desse período, constatando-se um aumento de 4,2 dias em relação ao café, onde se obteve o menor tempo de desenvolvimento (Tabela 1). Todavia, ambos os valores foram inferiores aos obtidos por CORREA et al. (2005) quando avaliaram o desenvolvimento de $P$. citri criada em folhas de citros (C. sinensis cv. Bahia) (31,2 dias).

Para a fase adulta de $P$. minor proveniente de cacau, os substratos café e citros promoveram um maior período de sobrevivência das fêmeas em relação ao cacau, permitindo cerca de 30 dias a mais de vida (Tabela 1). Para os machos, somente o citros provocou um prolongamento significativo desse período, todavia, nos três tratamentos, a longevidade foi próxima àquela encontrada por outros autores, como MALLESHAIAH et al. (2000) (4,4 dias) e CoRrea et al. (2005) (2,0 dias), trabalhando com a cochonilha $P$. citri criada em frutos de abóbora e folhas de citros, respectivamente.

O ciclo de vida (período ninfal + longevidade) de P. minor oriunda de plantas de cacau foi influenciado pelos substratos alimentares, sendo que as fêmeas mantidas em café e citros, e os machos criados em citros, apresentaram um maior tempo de vida (Tabela 1).

O desenvolvimento ninfal de fêmeas provenientes de cafeeiro sofreu influência dos hospedeiros sobre os quais foram criadas (Tabela 2), sendo que o substrato café se apresentou como o mais favorável, por permitir menor duração desse período. O citros acarretou a maior duração, constatando-se um prolongamento de 5,8 dias em relação ao café, resultado muito próximo ao obtido por CORREA et al. (2005) para P. citri em citros (cv. Bahia) (24,3 dias). Os valores constatados para a duração desse período nos hospedeiros café e cacau se aproximaram daqueles verificados por BISWAS; GHOSH (2000), que estudaram 
a biologia da cochonilha P. minor em soja (17,5 dias), ixora (Ixora signaporensis) (16,2 dias) e rabo de gato (Acalypha wilkesiana) (21,8 dias).

Os substratos alimentares apresentaram um efeito semelhante ao verificado para as fêmeas sobre a duração do período ninfal de machos de $P$. minor provenientes de plantas de café (Tabela 2). Da mesma forma, o substrato café proporcionou o menor tempo de desenvolvimento, enquanto que o citros foi o que acarretou maior duração até atingir a fase adulta. O período ninfal constatado para os machos criados em café foi similar aos verificados por CoRrea et al. (2006) para P. citri mantida nos cultivares de cafeeiro Acaiá Cerrado (22,3 dias) e Apoatã (20,2 dias), contudo, discreparam daqueles obtidos por Laflin; Parrella (2004) em rosas $(36,6$ dias). Essa discrepância provavelmente se deve ao efeito da temperatura, haja vista os experimentos terem sido conduzidos em torno de $20^{\circ} \mathrm{C}$, ou mesmo a um efeito do substrato alimentar.

Tabela 1 - Duração média (dias) ( \pm erro padrão) do desenvolvimento de fêmeas e machos de Planococcus minor (Hemiptera: Pseudococcidae) oriundos de cacaueiro e criados em folhas de diferentes hospedeiros, $\left(25 \pm 1^{\circ} \mathrm{C}, 70 \pm 10 \%\right.$ UR e 12 horas de fotofase).

\begin{tabular}{|c|c|c|c|c|}
\hline \multirow{3}{*}{ Fase } & \multicolumn{3}{|c|}{ Hospedeiros } & \multirow{3}{*}{ Valor $\mathrm{P}^{*}$} \\
\hline & \multirow{2}{*}{$\begin{array}{c}\text { cacau } \\
\text { Theobroma cacao }\end{array}$} & \multirow{2}{*}{$\begin{array}{c}\text { café } \\
\text { Coffea arabica }\end{array}$} & \multirow{2}{*}{$\begin{array}{c}\text { citros } \\
\text { Citrus sinensis }\end{array}$} & \\
\hline & & & & \\
\hline Período ninfal & $24,3 \pm 1,9$ & $23,9 \pm 1,4$ & $23,5 \pm 1,0$ & 09558 \\
\hline$(\mathrm{F})$ & $\mathrm{n}=3$ & $\mathrm{n}=14$ & $\mathrm{n}=11$ & 0,9050 \\
\hline Período ninfal & $23,0 \mathrm{ab}$ & $21,8 \pm 0,6 b$ & $26,0 \pm 1,1 \mathrm{a}$ & 0,003 \\
\hline (M) & $\mathrm{n}=1$ & $\mathrm{n}=24$ & $\mathrm{n}=12$ & 0,000 \\
\hline Longevidade & $21,3 \pm 6,9 b$ & $53,8 \pm 4,8 \mathrm{a}$ & $51,1 \pm 2,8 \mathrm{a}$ & 0,002 \\
\hline (F) & $\mathrm{n}=3$ & $\mathrm{n}=14$ & $\mathrm{n}=11$ & 0,002 \\
\hline Longevidade & $3,0 \mathrm{ab}$ & $2,3 \pm 0,2 b$ & $3,2 \pm 0,3 \mathrm{a}$ & \\
\hline$(\mathrm{M})$ & $\mathrm{n}=1$ & $\mathrm{n}=24$ & $\mathrm{n}=12$ & 0,05 \\
\hline Ciclo de vida & $45,7 \pm 5,0 b$ & $77,6 \pm 5,0 \mathrm{a}$ & $74,6 \pm 2,7 a$ & 0.005 \\
\hline$(\mathrm{F})$ & $\mathrm{n}=3$ & $\mathrm{n}=14$ & $\mathrm{n}=11$ & 0,000 \\
\hline Ciclo de vida & $26,0 \mathrm{ab}$ & $24,1 \pm 0,6 \mathrm{~b}$ & $29,2 \pm 1,1 \mathrm{a}$ & $<0.001$ \\
\hline (M) & $\mathrm{n}=1$ & $\mathrm{n}=24$ & $\mathrm{n}=12$ & 0,001 \\
\hline
\end{tabular}

*Análise de variância com dados transformados por $\sqrt{x}$. Médias seguidas com mesma letra nas linhas não diferem entre si pelo teste de Tukey a $5 \%$.

$\mathrm{F}=$ fêmea, $\mathrm{M}=$ macho; $\mathrm{n}$ = número de indivíduos observados.

Tabela 2 - Duração média (dias) ( \pm erro padrão) do desenvolvimento de fêmeas e machos de Planococcus minor (Hemiptera: Pseudococcidae) oriundos de cafeeiro e criados em folhas de diferentes hospedeiros, $\left(25 \pm 1^{\circ} \mathrm{C}, 70 \pm 10 \%\right.$ UR e 12 horas de fotofase).

\begin{tabular}{|c|c|c|c|c|}
\hline \multirow{3}{*}{ Fase } & \multicolumn{3}{|c|}{ Hospedeiros } & \multirow{3}{*}{ Valor $\mathrm{P}^{*}$} \\
\hline & cacau & café & citros & \\
\hline & Theobroma cacao & Coffea arabica & Citrus sinensis & \\
\hline $\begin{array}{l}\text { Período ninfal } \\
\text { (F) }\end{array}$ & $\begin{array}{c}19,5 \pm 0,5 a b \\
n=2\end{array}$ & $\begin{array}{c}19,2 \pm 0,6 b \\
n=24\end{array}$ & $\begin{array}{c}25,0 \pm 1,7 \mathrm{a} \\
\mathrm{n}=4\end{array}$ & 0,003 \\
\hline $\begin{array}{l}\text { Período ninfal } \\
\text { (M) }\end{array}$ & $\begin{array}{c}23,7 \pm 1,8 \mathrm{ab} \\
\mathrm{n}=3\end{array}$ & $\begin{array}{c}21,3 \pm 0,4 b \\
n=20\end{array}$ & $\begin{array}{c}25,5 \pm 0,9 a \\
n=4\end{array}$ & 0,002 \\
\hline $\begin{array}{l}\text { Longevidade } \\
\text { (F) }\end{array}$ & $\begin{array}{c}40,5 \pm 12,5 a b \\
n=2\end{array}$ & $\begin{array}{c}59,1 \pm 2,8 a \\
n=24\end{array}$ & $\begin{array}{c}37,5 \pm 6,9 b \\
n=4\end{array}$ & 0,007 \\
\hline $\begin{array}{l}\text { Longevidade } \\
\text { (M) }\end{array}$ & $\begin{array}{c}3,0 \pm 0,0 \\
n=3\end{array}$ & $\begin{array}{c}2,7 \pm 0,2 \\
n=20\end{array}$ & $\begin{array}{c}2,3 \pm 0,3 \\
n=4\end{array}$ & 0,530 \\
\hline $\begin{array}{l}\text { Ciclo de vida } \\
\text { (F) }\end{array}$ & $\begin{array}{c}60,0 \pm 13,0 b \\
n=2\end{array}$ & $\begin{array}{c}78,3 \pm 2,8 a \\
n=24\end{array}$ & $\begin{array}{c}62,5 \pm 6,4 \mathrm{ab} \\
\mathrm{n}=4\end{array}$ & 0,039 \\
\hline $\begin{array}{l}\text { Ciclo de vida } \\
(\mathrm{M})\end{array}$ & $\begin{array}{c}26,7 \pm 1,8 \mathrm{ab} \\
\mathrm{n}=3\end{array}$ & $\begin{array}{c}23,9 \pm 0,5 b \\
n=20\end{array}$ & $\begin{array}{c}27,8 \pm 0,6 \mathrm{a} \\
\mathrm{n}=4\end{array}$ & 0,005 \\
\hline
\end{tabular}

*Análise de variância com dados transformados por $\sqrt{x}$. Médias seguidas com mesma letra nas linhas não diferem entre si pelo teste de Tukey a $5 \%$.

$\mathrm{F}=$ fêmea, $\mathrm{M}$ = macho; $\mathrm{n}$ = número de indivíduos observados. 
Alongevidade dasfêmeas provenientes de plantas de café diferiu em função dos substratos alimentares avaliados, com uma variação de 21,6 dias entreaquelas criadas em café e citros, verificando-se que a duração desse período foi ampliada quando criadas em café (Tabela 2). O tempo de vida das fêmeas que se desenvolveram em citros foi reduzido em 18,9 dias em relação ao resultado de CORREA et al. (2005), obtido para P. citricriada na cultivar Bahia. Tais divergências podem estar relacionadas ao uso de cultivares diferentes. Segundo BERNAYS; CHAPMAN (1994), a seleção do hospedeiro não envolve somente a escolha correta da espécie de planta, mas também a seleção de uma planta individual dentro da espécie que é suscetível para alimentação, sobrevivência e desenvolvimento.

O período de vida adulta dos machos não foi afetado pelo hospedeiro em que se desenvolveram. Com relação ao ciclo de vida, verificou-se que os hospedeiros exerceram influência sobre o desenvolvimento desses insetos. As fêmeas apresentaram um ciclo mais longo quando criadas em café, e os machos, quando criados em citros (Tabela 2).

A duração do período ninfal de fêmeas provenientes de citros foi muito influenciada pelos substratos (Tabela 3), visto que o cacau exerceu um efeito deletério sobre elas, não permitindo que nenhuma atingisse a fase adulta. Uma maior velocidade de desenvolvimento foi obtida quando esses insetos foram criados em café, em relação aqueles criados em citros. Para os machos, não houve influência dos hospedeiros sobre a duração da fase de ninfa. Correa et al. (2006), estudando a biologia de P. citri em cafeeiros, encontraram valores muito próximos para a duração desse período para fêmeas criadas nos cultivares Acaiá Cerrado (20,2 dias) e Mundo Novo (23,6 dias). MARTínez; SuRís (1998) obtiveram resultados semelhantes para o período ninfal de machos (21,3 dias) para P. minor criada em batata.

A duração do período de vida adulta das fêmeas de $P$. citri não foi afetada pelos substratos sobre os quais foram alimentadas, enquanto os machos apresentaram um prolongamento dessa fase quando criados em citros (Tabela 3). Porém, o tempo de vida dos machos se assemelhou aos verificados em outros trabalhos, como aqueles conduzidos por Correa (2006) e Malleshaiah et al. (2000) para P. citri, em cafeeiro e frutos de abóbora, respectivamente. Quando se avaliou o ciclo de vida desses insetos não foram verificadas diferenças no tempo de desenvolvimento de fêmeas e machos em função dos hospedeiros estudados (Tabela 3).

Os substratos alimentares exerceram influência na mortalidade das ninfas de Planococcus spp. oriundas dos três hospedeiros (Tabela 4). Os maiores índices de mortalidade foram obtidos quando criadas no substrato cacau, independente da planta hospedeira da qual foram originalmente coletadas, e quando mantidas em citros, a partir de insetos coletados em plantas de café. Nessas condições, os valores de mortalidade foram superiores a $80 \%$. As menores porcentagens de mortalidade foram encontradas quando as ninfas se desenvolveram no substrato café, independente da procedência hospedeira, e no citros, quando oriundas de plantas de citros.

Tabela 3 - Duração média (dias) ( \pm erro padrão) do desenvolvimento de fêmeas e machos de Planococcus citri (Hemiptera: Pseudococcidae) oriundos de citros e criados em folhas de diferentes hospedeiros, $\left(25 \pm 1^{\circ} \mathrm{C}, 70 \pm 10 \%\right.$ UR e 12 horas de fotofase).

\begin{tabular}{|c|c|c|c|c|}
\hline \multirow{3}{*}{ Fase } & \multicolumn{3}{|c|}{ Hospedeiros } & \multirow{3}{*}{ Valor $\mathrm{P}^{*}$} \\
\hline & cacau & café & citros & \\
\hline & Theobroma cacao & Coffea arabica & Citrus sinensis & \\
\hline $\begin{array}{l}\text { Período ninfal } \\
\text { (F) }\end{array}$ & $* *$ & $\begin{array}{c}20,1 \pm 0,9 b \\
n=20\end{array}$ & $\begin{array}{c}23,4 \pm 0,8 a \\
n=10\end{array}$ & 0,016 \\
\hline $\begin{array}{l}\text { Período ninfal } \\
(\mathrm{M})\end{array}$ & $\begin{array}{c}22,3 \pm 0,7 \\
n=6\end{array}$ & $\begin{array}{c}20,0 \pm 0,6 \\
n=29\end{array}$ & $\begin{array}{c}20,3 \pm 0,3 \\
n=28\end{array}$ & 0,122 \\
\hline $\begin{array}{l}\text { Longevidade } \\
(\mathrm{F})\end{array}$ & ** & $\begin{array}{c}55,5 \pm 4,1 \\
n=20\end{array}$ & $\begin{array}{c}51,5 \pm 2,9 \\
n=10\end{array}$ & 0,656 \\
\hline $\begin{array}{l}\text { Longevidade } \\
\text { (M) }\end{array}$ & $\begin{array}{c}2,3 \pm 0,3 b \\
n=6\end{array}$ & $\begin{array}{c}2,8 \pm 0,1 \mathrm{ab} \\
\mathrm{n}=29\end{array}$ & $\begin{array}{c}3,1 \pm 0,1 a \\
n=28\end{array}$ & 0,010 \\
\hline $\begin{array}{l}\text { Ciclo de vida } \\
\text { (F) }\end{array}$ & $* *$ & $\begin{array}{c}75,6 \pm 4,2 \\
n=20\end{array}$ & $\begin{array}{c}74,9 \pm 3,0 \\
n=10\end{array}$ & 0,969 \\
\hline $\begin{array}{l}\text { Ciclo de vida } \\
\text { (M) }\end{array}$ & $\begin{array}{c}24,7 \pm 1,0 \\
n=6\end{array}$ & $\begin{array}{c}22,8 \pm 0,6 \\
n=29\end{array}$ & $\begin{array}{c}23,3 \pm 0,4 \\
n=28\end{array}$ & 0,286 \\
\hline
\end{tabular}


Tabela 4 - Mortalidade média (\%) de ninfas (fêmeas e machos) de Planococcus spp. (Hemiptera: Pseudococcidae) provenientes de diferentes origens e criadas em três hospedeiros, $\left(25 \pm 1^{\circ} \mathrm{C}, 70 \pm 10 \%\right.$ UR e 12 horas de fotofase).

\begin{tabular}{ll}
\hline Origem/hospedeiros & Período ninfal \\
\hline P. minor [Cacaueiro (Theobroma cacao)] & $93,4 \mathrm{a}$ \\
\hline cacau & $36,7 \mathrm{c}$ \\
café & $61,7 \mathrm{~b}$ \\
\hline citros & $90,0 \mathrm{a}$ \\
\hline$P$. minor [Cafeeiro (Coffea canephora)] & $26,7 \mathrm{~cd}$ \\
\hline cacau & $86,7 \mathrm{a}$ \\
café & $90,0 \mathrm{a}$ \\
citros & $18,3 \mathrm{~d}$ \\
\hline$P$. citri [Cítricos (Citrus sinensis)] & $36,7 \mathrm{c}$ \\
\hline cacau & $<0,001$ \\
café & $<$ \\
citros & \\
\hline Valor P* & \\
${ }^{*}$ Médias seguidas com mesma letra na coluna não diferem entre si pelo Teste de Qui-quadrado $\left(\chi^{2}\right)$ a $5 \%$. \\
$* *$ Para fins de estudo, a mortalidade média foi considerada alta quando seguidas pelas letras a e b, e baixa seguidas \\
pelas c e d.
\end{tabular}

Vários autores, estudando a biologia de pseudococcídeos, obtiveram índices de mortalidade baixos, como GARCIA et al. (1992), que estudaram a cochonilha Dysmicoccus cryptus Hempel, 1918, em tubérculos de batata (3\%); CorREA et al. (2005) que estudaram P. citri em folhas de citros (30\%) e Correa (2006) quando estudou essa mesma espécie em cultivares de café (abaixo de 30\%).

De uma maneira geral, os discos foliares de cacau se mostraram menos adequados para as diferentes populações de cochonilhas, pois, embora não tenham prolongado o período de desenvolvimento ninfal, proporcionaram as maiores taxas de mortalidade, além de terem causado a morte de todas as ninfas fêmeas oriundas de plantas de citros (Tabela 3). Esse fato fornece subsídios para se sugerir que folhas de cacau apresentam alguma particularidade que afeta negativamente o desenvolvimento dessas cochonilhas. Defato, como relatado por PIzZAMiglio (1991), as plantas desenvolvem estruturas físicas e/ou substâncias químicas que causam efeitos deletérios aos insetos não adaptados a se alimentar delas. Esse efeito no metabolismo do inseto pode ser decorrente principalmente da ingestão de metabolitos tóxicos, inibidores enzimáticos e reprodutivos, ou da deficiência qualitativa e quantitativa de nutrientes.

As cochonilhas que foram criadas no substrato cacau apresentaram-se com tamanho menor em comparação com os insetos criados nos demais substratos. De acordo com Evans (1984), as deficiências dietéticas podem causar a redução no tamanho e funcionamento das glândulas endócrinas, resultando em desenvolvimento anormal e, em casos extremos, podem causar inanição ou morte. Entretanto, populações de P. minor foram observadas em densidades relativamente elevadas sobre plantas de cacau cultivadas em casa de vegetação. Além disso, existem relatos da ocorrência desses insetos em lavouras de cacau da região Neotropical e regiões da Polinésia, Australo-oriental e Oriental (COx; FREESTON, 1985).

Essas constatações levam a sugerir a existência de uma especialização do inseto, pois a maioria das espécies fitófagas tendea especializar-secom o táxon da planta da qual se alimentam, podendo também se associar com partes particulares das plantas (BERNAYS; CHAPMAN, 1994). Como os espécimes utilizados neste experimento foram originalmente coletados em frutos de cacaueiros, eles podem ter sofrido o efeito, não apenas da mudança de hospedeiro, mas também da alteração da parte da planta, ou seja, dos frutos para os discos foliares. Esse fenômeno também foi observado por KirKPATRICK (1953) que, ao estudar cochonilhas (Planococcus sp.) presentes em cacaueiros de Trinidad, verificou que elas podiam se desenvolver sobre frutos de citros, mas não em folhas.

Osubstrato café apresentou-se como o mais satisfatório para o desenvolvimento das cochonilhas, uma vez que proporcionou as maiores taxas de sobrevivência e, de maneira geral, reduziu o período de ninfa de fêmeas e machos. Além disso, permitiu uma maior longevidade desses insetos, sugerindo que esse hospedeiro provém condições mais favoráveis para seu desenvolvimento. Segundo PARRA (1991), a qualidade e a quantidade do alimento ingerido na fase jovem afetam a taxa de crescimento, o tempo de desenvolvimento, peso do corpo, sobrevivência, bem como influenciam a fecundidade, longevidade, movimentação e capacidade de competição de adultos. Assim, dietas inadequadas induzem o inseto a prolongar o desenvolvimento ninfal. 
Oprolongamento do tempo de vida adulta provocado pelo substrato caféfortalece o indício de tratar-se de um alimento adequado, pois o desempenho de cada estágio de vida do inseto depende basicamente do sucesso do estágio anterior em obter, sintetizar e acumular as substâncias nutricionais em quantidades apropriadas (PARRA, 1991).

Quanto à busca de uma especificidade alimentar dessas populações decochonilhas dogêneroPlanococcus, pode-se relatar, baseando-se nos resultados obtidos para sobrevivência e duração dos estágios de vida, que as populações provenientes de cacaueiros e cafeeiros ( $P$. minor) se desenvolvem melhor em café, e que as oriundas de citros (P. citri) em café e citros. Dessa forma, foi observado maior preferência alimentar de $P$. minor para o substrato café, independente do seu hospedeiro de origem, e maior preferência alimentar de $P$. citri para café e citros, indicando uma especificidade parcial destas espécies de cochonilhas.

$\mathrm{O}$ fato de se ter constatado uma especificidade hospedeira parcial entre as populações de cochonilhas do gênero Planococcus, permite sugerir que plantas de cacau são infestadas preferencialmente por $P$. minor e plantas de citros preferencialmente por $P$. citri. Por outro lado, plantas de café poderão ser infestadas por $P$. minor e $P$. citri.

\section{CONCLUSÃO}

Populações da cochonilha P. minor provenientes de plantas de café e cacau apresentam preferência alimentar para folhas de cafeeiro. Enquanto que populações de $P$. citri oriundas de plantas de citros apresentam preferência alimentar para café e citros. Isto indica uma especificidade hospedeira parcial para estas espécies de cochonilhas.

\section{REFERÊNCIAS}

BERNAYS, E.A.; CHAPMAN, R.F. Host-plant selection by phytophagous insects. New York: Chapman \& Hall, 1994. $312 \mathrm{p}$.

BISWAS, J.; GHOSH, A.B. Biology of the Mealybug, Planococcus minor (Maskell) on various host plants. Environment and Ecology, v.18, n.4, p.929-932, 2000.

CLAPS, L.E.; TERÁN, A.L. Diaspididae (Hemiptera: Coccoidea) asociadas a cítricos en la Provincia de Tucumán (Republica da Argentina). Neotropical Entomology, v.30, n.3, p.391-402, 2001.

CORREA, L.R.B.; BONANI, J.P.; SANTA-CECÍLIA, L.V.C.; SOUZA, B. Aspectos biológicos da cochonilhabranca [Planococcus citri (Risso, 1813)] em citros. Laranja, v.26, n.2, p.265-271, 2005.
CORREA, L.R.B. Efeitos de diferentes temperaturas e substratos alimentares sobre o desenvolvimento da cochonilhabranca Planococcus citri (Risso, 1813) (Hemiptera: Pseudococcidae). 2006. 16f. Monografia (Graduação em Agronomia - Área Entomologia) - Universidade Federal de Lavras, Lavras, MG.

CORREA, L.R.B.; SANTA-CECÍLIA, L.V.C.; PRADO, E.; SOUZA, B.; ALCANTRA, E. Desenvolvimento da cochonilha-branca Planococcus citri (Risso, 1813) (Hemiptera: Pseudococcidae) em cafeeiros. In: SEMINÁRIO DE INICAÇÃO CIENTÍFICA E TECNOLOGICA, 3., 2006, Belo Horizonte. Anais. Belo Horizonte: EPAMIG, 2006. 1-CDROM.

COX, J.M. An experimental study of morphological variation in mealybugs (Homoptera: Coccoidea: Pseudococcidae). Systematic Entomology, v.8, p.361-382, 1983.

COX, J.M. The mealybug genus Planococcus (Homoptera: Pseudococcidae). Bulletin of the British Museum Natural History Entomology, v.58, n.1, p.1-78, 1989.

COX, J.M.; FREESTON, A.C. Identification of mealybugs of the genus Planococcus (Homoptera: Pseudococcidae) occurring on cacao throughout the world. Journal of Natural History, v.19, p.719-728, 1985.

EVANS, H.E. Insect biology. Massachusetts: AddisonWesley, 1984. 436p.

GARCIA, A.; ALAUZET, C.; DECAZY, B. Biologie de la cochonilhe racinaire du caféier Dysmicoccus cryptus (Hempel, 1918) comb.n. (Homoptera: Pseudococcidae). Café Cacao Thé, v.36, n.1, p.35-44, 1992.

HAGEN, K.S.; DADD, R.H.; REESE, J. The food of insects. In: HUFFAKER, C.B.; RABB, R.L. (Ed.). Ecological Entomology. New York: J. Wiley \& Sons, 1984. p.79-112.

KIRKPATRICK, T.W. Notes on minor insect pests of cacao in Trinidad. Report on Cacao Research, p.62-71, 1953

LAFLIN, H.M.; PARRELLA, M.P. Developmental biology of citrus mealybug under conditions typical of California rose production. Arthropod Biology, v.97, n.5, p.982- 988, 2004

MALLESHAIAH, B.; RAJAGOPAL, K.; GOWDA, K.N.M. Biology of citrus mealybug, Planococcus citri (Risso) (Hemiptera: Pseudococcidae). Crop Research, v.20, n.1, p.130-133, 2000.

MAITY, D.K.; SAHOO, A.K.; MANDAL, S.K. Evaluation of laboratory hosts for rearing and mass multiplication of Planococcus minor (Maskell) (Pseudococcidae: Hemiptera). Environment and Ecology, v.16, n.3, p.530-532, 1998.

MARTÍNEZ, M.A.; SURIS, M. Biology of Planococcus minor Maskell (Homoptera: Pseudococcidae) em condi- 
ciones de laboratorio. Revista de Protection Vegetal, v.13, n.3, p.199-201, 1998.

MILLER, D.R.; KOSZTARAB, M. Recent advances in the study of scales insects. Annual Review of Entomology, v.24, p.1-27, 1979.

NUR, U. Electrophoretic comparison of enzymes of sexual and parthenogenetic mealybugs. Virginia. Polytechnic. Institute State University Research Division Bull., v.127, p.69-84, 1978.

OSBORNE, D.J. Mutual regulation of growth and development in plants insects. In: VAN EMDEN, H.F. (Ed.). Insect/Plant Relationships. London: Oxford Blackwell, 1973. p.33-42.

PANIZZI, A.; PARRA, J.R. Ecologia nutricional e o Manejo Integrado de Pragas. In:

(Ed.). Ecologia nutricional de insetos e suas implicações no manejo de pragas. São Paulo: Manole, 1991. p.313-335.

PARRA, J.R. Consumo e utilização de alimentos por insetos. In: PANIZZI, A.; PARRA, J.R. (Ed.). Ecologia nutricional de insetos e suas implicações no manejo de pragas. São Paulo: Manole, 1991. p.9-65.
PIZZAMIGLIO, M.A. Ecologia das Interações Inseto Plantas. In: PANIZZI, A.; PARRA, J.R. (Ed.). Ecologia nutricional de insetos e suas implicações no manejo de pragas. São Paulo: Manole, 1991. p.101-129.

SANTA-CECÍLIA, L.V.C.; SOUZA, B. Controle biológico de cochonilhas-farinhentas em cultivos protegidos. Informe Agropecuário, v.26, n.225, p.24-30, 2005.

SANTA-CECÍLIA, L.V.C.; REIS, P.R.; SOUZA, J.C. Sobre a nomenclatura das espécies de cochonilhasfarinhentas do cafeeiro nos Estados de Minas Gerais e Espírito Santo. Neotropical Entomology, v.31, n.2, p.333334, 2002.

SANTA-CECÍLIA, L.V.C.; BUENO, V.H.P.; PRADO, E. Desenvolvimento de Dysmicoccus brevipes (Cockerell) (Hemiptera: Pseudococcidae) em duas cultivares de abacaxi. Ciência e Agrotecnologia, v.28, n.5, p.1015-1020, 2004.

SLANSKY JUNIOR, F.; RODRIGUEZ, J.G. (Ed.). Nutritional Ecology of insects, mites, spiders, and related invertebrates. New York: J. Wiley \& Sons, 1987. 955p.

Recebido em 13/3/09

Aceito em 19/1/11 